\title{
G6PD Polymorphisms and Hemolysis After Antimalarial Treatment With Low Single-Dose Primaquine: A Pooled Analysis of Six African Clinical Trials
}

\section{OPEN ACCESS}

Edited by:

Rajeev Kumar Mehlotra, Case Western Reserve University,

United States

Reviewed by:

J. Kevin Baird,

University of Oxford, United Kingdom Xi Wu,

Shanghai Jiao Tong University, China

*Correspondence: Chris Drakeley chris.drakeley@lshtm.ac.uk Nuno Sepúlveda nunosep@gmail.com

Specialty section: This article was submitted to Pharmacogenetics and Pharmacogenomics, a section of the journal

Frontiers in Genetics

Received: 23 December 2020 Accepted: 23 February 2021

Published: 09 April 2021

Citation:

Sepúlveda N, Grignard L, Curry J, Mahey L, Bastiaens GJH, Tiono $A B$, Okebe J, Coulibaly SA, Gonçalves BP, Affara M, Ouédraogo A, Bougouma EC, Sanou GS, Nébié l,

Lanke K, Sirima SB, Dicko A, d'Alessandro U, Clark TG,

Campino S, Chen I, Eziefula AC,

Gosling R, Bousema T and

Drakeley C (2021) G6PD

Polymorphisms and Hemolysis After Antimalarial Treatment With Low Single-Dose Primaquine: A Pooled Analysis of Six African Clinical Trials.

Front. Genet. 12:645688.

doi: 10.3389/fgene.2021.645688
Nuno Sepúlveda ${ }^{1,2 *}$, Lynn Grignard1', Jonathan Curry ${ }^{3}$, Laleta Mahey ${ }^{3}$ Guido J. H. Bastiaens ${ }^{4}$, Alfred B. Tiono ${ }^{5}$, Joseph Okebe ${ }^{6}$, Sam A. Coulibaly5, Bronner P. Gonçalves ${ }^{1,7}$, Muna Affara ${ }^{8}$, Alphonse Ouédraogo ${ }^{5}$, Edith C. Bougouma ${ }^{5}$, Guillaume S. Sanou ${ }^{5}$ Issa Nébié ${ }^{5}$, Kjerstin Lanke ${ }^{4}$, Sodiomon B. Sirima ${ }^{5}$, Alassane Dicko9 ${ }^{9}$, Umberto d'Alessandro ${ }^{8,10}$, Taane G. Clark ${ }^{1,7}$, Susana Campino1, Ingrid Chen ${ }^{11}$, Alice C. Eziefula ${ }^{1,12}$, Roly Gosling ${ }^{11}$, Teun Bousema ${ }^{1,4}$ and Chris Drakeley ${ }^{1 *}$

'Department of Infection Biology, Faculty of Infectious and Tropical Diseases, London School of Hygiene and Tropical Medicine, London, United Kingdom, ${ }^{2}$ CEAUL - Centro de Estatística e Aplicações da Universidade de Lisboa, Lisbon, Portugal, ${ }^{3}$ LGC Genomics, Hertfordshire, United Kingdom, ${ }^{4}$ Department of Medical Microbiology, Radboud University Medical Center, Nijmegen, Netherlands, ${ }^{5}$ Department of Public Health, Centre National de Recherche et de Formation sur le Paludisme \& Institut National de Santé Publique, Ouagadougou, Burkina Faso, ${ }^{6}$ Department of International Public Health, Liverpool School of Tropical Medicine, Liverpool, United Kingdom, ${ }^{7}$ Department of Infectious Disease Epidemiology, Faculty of Epidemiology and Population Health, London School of Hygiene \& Tropical Medicine, London, United Kingdom, ${ }^{8}$ MRC Unit The Gambia at the London School of Hygiene \& Tropical Medicine, Fajara, The Gambia, ${ }^{9}$ Malaria Research and Training Centre, Faculty of Pharmacy and Faculty of Medicine and Dentistry, University of Sciences, Techniques and Technology of Bamako, Bamako, Mali, ${ }^{10}$ Department of Disease Control, Faculty of Infectious and Tropical Diseases, London School of Hygiene \& Tropical Medicine, London, United Kingdom, "11 Department of Epidemiology and Biostatistics, University of California, San Francisco, San Francisco, CA, United States, ${ }^{12}$ Department of Global Health and Infection, Brighton and Sussex Medical School, University of Sussex, Brighton, United Kingdom

Primaquine $(P Q)$ is an antimalarial drug with the potential to reduce malaria transmission due to its capacity to clear mature Plasmodium falciparum gametocytes in the human host. However, the large-scale roll-out of PQ has to be counterbalanced by the additional risk of drug-induced hemolysis in individuals suffering from Glucose6-phospate dehydrogenase (G6PD) deficiency, a genetic condition determined by polymorphisms on the X-linked G6PD gene. Most studies on G6PD deficiency and $P Q$-associated hemolysis focused on the G6PD A-variant, a combination of the two single nucleotide changes G202A (rs1050828) and A376G (rs1050829), although other polymorphisms may play a role. In this study, we tested the association of 20 G6PD single nucleotide polymorphisms (SNPs) with hemolysis measured seven days after low single dose of $P Q$ given at the dose of $0.1 \mathrm{mg} / \mathrm{kg}$ to $0.75 \mathrm{mg} / \mathrm{kg}$ in 957 individuals from 6 previously published clinical trials investigating the safety and efficacy of this drug spanning five African countries. After adjusting for inter-study effects, age, gender, baseline hemoglobin level, PQ dose, and parasitemia at screening, our analysis showed putative association signals from the common G6PD mutation, A376G $\left[-\log _{10}(p-\right.$ value $)=2.44]$ and two less-known SNPs, rs2230037 [- $\log _{10}(D$-value] $=2.60)$, and rs28470352 [ $-\log _{10}(p$-value $\left.)=2.15\right] ; A 376 G$ and rs2230037 were in very strong linkage disequilibrium with each other $\left(R^{2}=0.978\right)$. However, when the effects of these SNPs 
were included in the same regression model, the subsequent associations were in the borderline of statistical significance. In conclusion, whilst a role for the A- variant is well established, we did not observe an important additional role for other G6PD polymorphisms in determining post-treatment hemolysis in individuals treated with low single-dose $P Q$.

Keywords: genetic association study, clinical trials, malaria, drug safety, hemoglobin

\section{INTRODUCTION}

The last two decades of intensive malaria control have achieved a significant reduction of malaria cases and deaths and, consequently, several endemic countries were officially declared in either pre-elimination, elimination, or eradication stages. One approach to accelerate disease elimination is to target the sexual and transmission stages of the parasite and, thereby, block transmission from humans to mosquitoes. The 8-aminoquinoline, Primaquine (PQ), can clear P. falciparum gametocytes and is typically used as a single-dose treatment. The drug at larger doses has also the ability to clear Plasmodium vivax and Plasmodium ovale hypnozoites. However, the biochemical action of the drug is known to induce transient hemolysis in treated individuals. The recognition of this side effect led to the evaluation of the efficacy and safety of treatments based on lower single doses of PQ for gametocyte clearance in 2011 (Eziefula et al., 2014). Later, the World Health Organisation (WHO) revised treatment guidelines and reduced the recommended PQ dose from 0.75 to $0.25 \mathrm{mg} / \mathrm{kg}$ in areas of $P$. falciparum elimination and/or high malaria drug resistance (WHO Malaria Policy Advisory Committee and Secretariat, 2012; World Health Organization, 2012). After this revision, several randomized controlled clinical trials and community studies were performed with the specific aim to assess both the efficacy and the safety of different PQ drug regimens, including the one based on the new recommended dose (Dicko et al., 2016; Gonçalves et al., 2016; Stone et al., 2017; Bastiaens et al., 2018; Chen et al., 2018).

The potential use of PQ-based treatments in large-scale malaria control and elimination campaigns might pose an additional risk to individuals with Glucose-6-phospate dehydrogenase (G6PD) deficiency (Baird and Surjadjaja, 2011; Awandu et al., 2018; Chen et al., 2018), a common red blood cell disorder in Africa (Howes et al., 2012). G6PD is a key enzyme in the pentose phosphate pathway that controls oxidative damage in erythrocytes. It is encoded by the G6PD gene, which is located in the telomeric region of the long arm of the $\mathrm{X}$ chromosome (Xq28). Since it results from an X-linked recessive transmission, G6PD deficiency is more frequent in males but effects are more difficult to predict in females due to random $\mathrm{X}$-chromosome inactivation. More than 150 single nucleotide

Abbreviations: BF1, dose-efficacy study from Burkina Faso; BF2, safety study from Burkina Faso; DBS, dried blood spots; DP, Dihydroartemisinin-piperaquine; FST, Fluorescence spot test; GAM, safety study from the Gambia; G6PD, Glucose6-phospate dehydrogenase; Hb, Hemoglobin; Hb0, Hemoglobin levels at day 0; Hb7, Hemoglobin levels at day 7; KEN, dose-efficacy study from Kenya; MAL, safety study from Mali; PQ, Primaquine; RDT, rapid diagnostic test; SNP, Single Nucleotide Polymorphism; UGD, dose-efficacy study from Uganda. polymorphisms (SNPs) have been identified in this gene with different impact on hemolysis and activity of the enzyme (Clarke et al., 2017). Additionally, there are specific variants that tend to confer protection against severe malaria-related anemia or cerebral malaria and these variants are under natural selection in malaria endemic countries (Maiga et al., 2014; Manjurano et al., 2015; Clarke et al., 2017; Sepúlveda et al., 2017). In Africa, the most important genetic markers for G6PD deficiency are the single nucleotide polymorphism (SNP) rs1050828 (G202A, or C202T, chrX:154,536,002) and rs1050829 (A376G, or T376C, chrX:154,535,277) (Rockett et al., 2014). A combination of these two single nucleotide changes (202A and 376G), also known as the A- variant, is associated with an $88 \%$ loss of G6PD enzyme activity (Hirono and Beutler, 1988; Beutler et al., 1989), which decreases the tolerance to 8-aminoquinolines. In principle, G6PD polymorphisms other than the classical A-variant should not be associated with significant hemolysis after a single-dose PQ treatment, but this expectation has never been tested with data from the field.

To fill in this research gap, we performed a genetic association analysis of 20 G6PD SNPs using data of 952 individuals from 6 PQ-related clinical trials in Africa: 3 dose-efficacy trials and 3 safety trials. These clinical trials were mostly conducted in G6PD-normal individuals. The subsequent findings aimed to contribute to a more accurate pharmacovigilance of PQtreatment in the continent.

\section{MATERIALS AND METHODS}

\section{Outline of Available PQ Clinical Trials}

Data from 957 individuals enrolled in six PQ clinical trials in Africa were made available for this analysis (Table 1): 330, 107, and 367 individuals from dose-efficacy studies performed in Burkina Faso (BF1), Kenya (KEN), and Uganda (UGD), respectively (Eziefula et al., 2014; Gonçalves et al., 2016; Stone et al., 2017); 77, 50, and 26 individuals from safety studies conducted in Burkina Faso (BF2), The Gambia (GAM), and Mali (MAL), respectively (Dicko et al., 2016; Bastiaens et al., 2018).

Briefly, only children were recruited in the BF1, KEN, and UGD dose-efficacy studies. With respect to the safety studies, only male adults were enrolled in BF2 and MAL whilst male adolescents were the majority of the participants from the GAM study. Only G6PD-normal individuals were considered eligible to participate in BF1, MAL, and UGD. In contrast, BF2 and GAM included both deficient and normal individuals with respect to G6PD enzymatic activity. In KEN, the G6PD status was not 
screened at enrollment. In all safety studies, PQ treatment was administrated together with a partner drug at day 0 of the trial. In the dose-efficacy studies, participants received a 3-day course of dihydroartemisinin-piperaquine (DP) alone or with a single low dose of PQ $(0.25 \mathrm{mg} / \mathrm{kg})$ on the third day of DP treatment (day 2 of the trial). For additional information about participant's recruitment, inclusion/exclusion criteria, and ethics, consult the original research protocol of each study.

\section{Measurement of Hemoglobin Levels and Parasitemia}

At enrollment (day 0) and on day 7 of the trials, all study participants had their hemoglobin $(\mathrm{Hb})$ concentration quantified (g/dL) and had a blood slide prepared to detect malaria parasites in 100 microscopic fields and quantified against 200 and 500 leukocytes, and then finally translated into parasite counts/ $\mu 1$ under the assumption of 8,000 leukocytes per $\mu l$.

\section{Diagnostics of G6PD Deficiency}

Participants were screened for G6PD deficiency at enrollment in all studies with the exception of KEN. The BinaxNOW rapid diagnostic test (RDT; Alere Inc., Waltham, MA, United States) was used in BF1 and Beutler's fluorescence spot test (FST R\&D Diagnostics, Greece) was used in all other studies where G6PD deficiency was screened.

\section{DNA Extraction and Genotyping}

DNA was extracted from dried blood spots (DBS) from UGD, KEN, and BF1 using the QIAamp DNA Mini Kit (Qiagen, United Kingdom). DNA was extracted from whole blood samples from GAM, MAL, and BF2 using the QIAamp DNA Blood Mini Kit (Qiagen, United Kingdom). All extractions were performed according to manufacturer's recommendations.

SNPs were genotyped using either the Kompetitive Allele Specific PCR (KASP) assay (LGC Genomics, United Kingdom) or a customized version of the High-throughput Mutation Screen kit for G6PD (Diacurate Inc., China). The KASP assay (Panel 1: GAM and BF1 and Panels 2: BF2, Supplementary Table 1) contains two allele-specific forward primers, one common reverse primer specific for each SNP, $0.8 \mu \mathrm{L}$ of genomic DNA suspension and $0.8 \mu \mathrm{L} 2 \times \mathrm{KASP}$ reaction mix. The Highthroughput Mutation Screen kit for G6PD consists of a multiplex extension and ligation-based probe amplification directly from DBS and was performed in China by Diacurate Incorporated. The customized assay interrogates 23 SNPs (Panel 3: KEN, MAL, and UGD, Supplementary Table 1). Following completion of the PCR, each sample was assigned a genotype based on cluster plot analysis of raw data using LGC's proprietary Kraken software.

\section{Genetic Data and Quality Controls}

To ensure high quality SNP data, one needs to perform routine quality checks for genetic association studies (Grabowska et al., 2020). In this regard, we excluded all SNPs from the analysis if these SNPs were monomorphic (three SNPs), had a minor allele frequency $<5 \%$ (seven SNPs), had a heterozygous frequency $>2 \%$ on males (one SNP), had a strong deviation from the
Hardy-Weinberg equilibrium on females (two SNPs; $p<0.001$; Chi-square goodness-of-fit tests), and had a frequency of missing data $>10 \%$ (thirteen SNPs). After these quality checks, there was evidence for a total of 20 high-quality SNPs to be used in the subsequent genetic association analysis. For a matter of comparison, allelic frequencies of African populations $(n=1,003$ alleles in total) were compiled from the 1000 Genome Project as available in the database dbSNP (Auton et al., 2015). All of these genetic markers were annotated according to the forward strand and the reference genome assembly GRCh38.

\section{Genetic Association Analysis}

A linear regression approach was applied to study the genetic association between the selected SNPs and the hemoglobin level measured on day 7 after PQ treatment. For statistical convenience, the hemoglobin level was log-transformed so that the resulting data would follow an approximate Normal distribution. The statistical assessment of a genetic association between a particular SNP and the phenotype of interest was based on the comparison between two nested regression models using Wilk's log-likelihood ratio test. The first model was a non-genetic linear regression model that included the following covariates: study index, gender, age, presentation parasitemia, and hemoglobin level, both day on presentation (hereafter considered as day 0 ). The second model is a genetic linear regression that extends the latter by additionally including a given genetic effect of SNP under analysis. For each SNP, five different genetic effects were included in the analysis: general effect (no specific parametric structure on the genotype effects), dominance/recessive (genotypes including the dominant allele had the same effect), additive (the effect of an individual specific allele in the outcome is additive), and heterosis (both homozygous genotypes have the same effect). To simplify the presentation of the results, statistical significance for the association between a given SNP and the outcome was assessed by the minimum $p$-value of all the five $p$-values comparing the non-genetic model and five genetic models for the SNP under analysis. To control for multiple testing, the Benjamini-Hochberg procedure was applied to all the minimum $p$-values in order to obtain an overall false discovery rate of $5 \%$ (Benjamini and Hochberg, 1995). This procedure was based on the following algorithm: (i) order the $p$-values from the largest to the smallest, (ii) calculate the largest $\mathrm{k}$ that satisfy $p_{k}<0.05 / k$ where $\mathrm{p}_{k}$ is the $k$-th ordered $p$-value; (iii) reject the null hypothesis for all ordered $p$-values lower than $p_{k}$. Statistically significant SNPs were then assessed in terms of their linkage disequilibrium (LD) using the correlation coefficient $\mathrm{R}^{2}$.

\section{RESULTS}

\section{Inter-Study Variations in Hemoglobin Levels and PQ Treatment Doses}

This study encompassed a total of 957 individuals distributed across six studies with different sample sizes (Table 1). With respect to PQ treatment, $\mathrm{BF} 1, \mathrm{BF} 2$, and $\mathrm{KEN}$ studies compared a placebo group to another group treated with a PQ dose 
TABLE 1 | Demographics of each PQ safety and dose-efficacy studies.

\begin{tabular}{|c|c|c|c|c|c|c|}
\hline Characteristics & BF1 & BF2 & GAM & KEN & MAL & UGD \\
\hline Reference & Gonçalves et al., 2016 & Bastiaens et al., 2018 & Bastiaens et al., 2018 & Stone et al., 2017 & Dicko et al., 2016 & Eziefula et al., 2014 \\
\hline Type of study & Dose-efficacy & Safety & Safety & Dose-efficacy & Safety & Dose-efficacy \\
\hline Sample size & 330 & 77 & 50 & 107 & 26 & 367 \\
\hline G6PD status & BinaxNOW RDT & Beutler's FST & Beutler's FST & $\mathrm{N} / \mathrm{A}$ & Beutler's FST & Beutler's FST \\
\hline Males (n,\%) & $170(51.5)$ & $77(100)$ & $50(100)$ & $59(55.1)$ & $26(100)$ & $183(49.9)$ \\
\hline Mean age (range) & $7.7(2-14)$ & $28.8(18-44)$ & $16.7(10-40)$ & $9.6(5-15)$ & $35.3(18-50)$ & $5.1(1-10)$ \\
\hline \multicolumn{7}{|c|}{ Primaquine dose $(n, \%)$} \\
\hline 0.00 (placebo) & $105(31.8)$ & $10(13.0)$ & $0(0.0)$ & $53(49.5)$ & $0(0.0)$ & $74(20.2)$ \\
\hline $0.10-0.40 \mathrm{mg} / \mathrm{kg}$ & 225 (68.2) & $67(87.0)$ & $50(100.0)$ & $54(50.5)$ & $5(19.2)$ & $201(54.8)$ \\
\hline $0.41-0.75 \mathrm{mg} / \mathrm{kg}$ & $0(0.0)$ & $0(0.0)$ & $0(0.0)$ & $0(0.0)$ & $21(80.8)$ & $92(25.1)$ \\
\hline \multicolumn{7}{|c|}{ Mean parasitemia (range, parasites $/ \mu \mathrm{l}$ ) } \\
\hline Day 0 & $5984.5(0-237986)$ & $373.8(0-3348)$ & $90.2(0-2384)$ & $1692.2(0-31360)$ & $0(0)$ & $69766.5(48-518180)$ \\
\hline Day 7 & $0.4(0-124)$ & $0(0-0)$ & $0(0-0)$ & $1.6(0-40)$ & $0(0)$ & $0(0-16)$ \\
\hline \multicolumn{7}{|c|}{ Mean hemoglobin (range, g/dL) } \\
\hline Day 0 & $11.5(6-14.3)$ & $14.2(11.4-17.5)$ & $13.1(11-17.2)$ & $12.0(9.6-14.8)$ & $15.0(13.3-17.5)$ & $11.2(8-15.4)$ \\
\hline Day 7 & $11.3(7.9-15.1)$ & $13.5(10.22-17.1)$ & $12.5(9.9-17.5)$ & $12.2(8.6-14.8)$ & $14.3(12-16.2)$ & $10.8(7.4-15.5)$ \\
\hline
\end{tabular}

between $0.1 \mathrm{mg} / \mathrm{kg}$ and $0.4 \mathrm{mg} / \mathrm{kg}$. The GAM and MAL studies only included a single treatment group with a 0.1$0.5 \mathrm{mg} / \mathrm{kg}$ PQ dose. The study from Uganda was the only one encompassing three treatment arms (placebo, 0.1-0.4 mg/kg and $0.41-0.75 \mathrm{mg} / \mathrm{kg}$ ). In MAL, only $P$. falciparum negative individuals were recruited to the study.

On the day of treatment (day 0), the mean $\mathrm{Hb}$ ranged from 11.2 to 15.0 (overall range $=6.0-17.5) \mathrm{g} / \mathrm{dL}$ (Table 1 ). Since the targeted populations and recruitment strategies differed across studies (e.g., male adults in MAL study versus male and female children in UGD study), an inter-study variation on mean (or median) Hb levels on day 0 was expected (Figure 1A). Another possible source of inter-study variation on $\mathrm{Hb}$ levels on day 0 ( $\mathrm{Hb} 0)$ was related to differences in parasitemia at presentation (Figure 1B). Hb0 was lower with the higher parasite counts in the blood (Spearman's correlation coefficient $=-0.37, p<0.001$ ). Unsurprisingly, PQ treatment was associated with a drop in the $\mathrm{Hb}$ levels at day 7 ( $\mathrm{Hb} 7)$ in the majority of the individuals (Figure 1C) with the level of reduction differing by study. The studies showed substantial heterogeneity in Hb levels, both on days 0 and 7 after PQ treatment.

When a non-genetic linear regression model was initially fitted to the data (Supplementary Table 2), there was evidence for a negative effect of parasitemia, a positive effect of age, and a positive effect of log-Hb0 on the log-Hb7 coefficient estimates $=-0.033,0.004$, and 0.567 , respectively, all $p$-values $<0.001)$. In addition, the effects of different studies on log- $\mathrm{Hb} 7$ were not statistically significant $(p>0.05)$ with the exception of the study from Kenya (coefficient estimate $=0.047, p<0.001$; Figure 1C). The effect of each PQ dose range was negative on the $\mathrm{Hb}$ levels at day 7 (coefficient estimates $=-0.010$ and -0.019 for $0.1-0.4$ and $>0.4 \mathrm{mg} / \mathrm{kg}$, respectively), which suggested some degree of hemolysis due to PQ treatment itself. However, these effects referring to PQ dose ranges were not statistically significant ( $p$-values $=0.195$ and 0.112 , respectively). Finally, this initial model including study index, gender, age, parasitemia at presentation, and $\mathrm{Hb} 0$ as covariates could explain $61 \%$ of the total variation observed in the log-Hb7 levels (adjusted $R^{2}=0.61$ ). We then investigated whether genetic variation on G6PD locus could explain the remaining random variation in the outcome.

\section{Analysis of G6PD SNPs}

Genetic analysis initiated by assessing the quality of the genotype data. Twenty out of the 23 initially genotyped SNPs were considered with high quality and, thus, they were carried forward to the association study (Supplementary Table 3). Of note, ten of the selected SNPs were located within the coding region of the G6PD gene. Two of these SNPs represented synonymous variants, other two referred to known missense variants with pathogenic potential (rs1050829 and rs1050828) and the remaining six SNPs were variants in the intronic regions of the G6PD gene. The other 10 SNPs were located in the flanking regions of $G 6 P D$, namely, in intronic regions of TLK1, RPL10, SNORA70, and IKBKG genes. Twelve of these SNPs could not be genotyped in the samples from BF2 due to unavailability of DNA material to genotype these SNPs.

In male participants, the allele frequency profile substantially differed from one study to another, specifically, in SNPs located within or very close to the coding region of G6PD (Table 2 and Figure 2A). The same finding was obtained when the allele frequencies associated with each study were compared to those available from the combined 1000 Genome Project for African populations (Figure 2B and Supplementary Figure 1). Interestingly, this variation became less obvious in SNPs located in the respective flanking regions, probably due to a decrease in linkage with variants within the G6PD locus.

In female participants, the allele frequency profile did not significantly differ between studies (Figure 2A). Importantly, the allelic frequency profile was consistent with the one available from the combined 1000 Genome project for African populations (Figure 2B). 


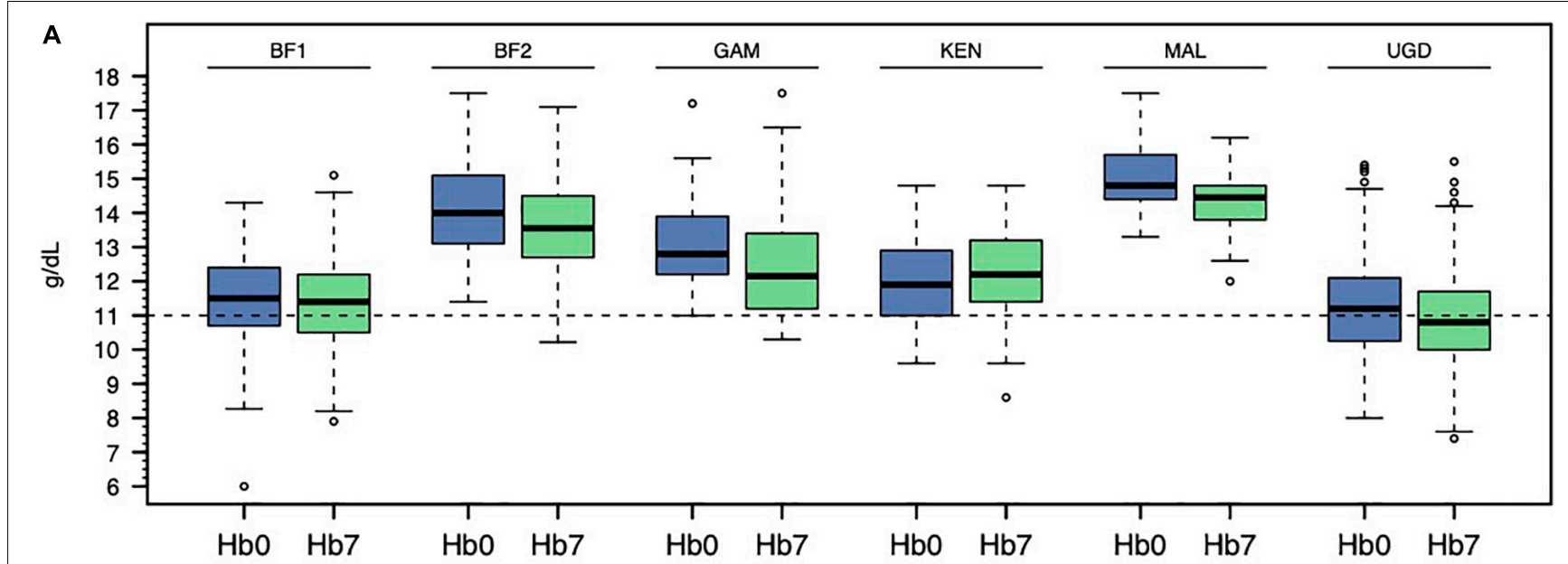

B

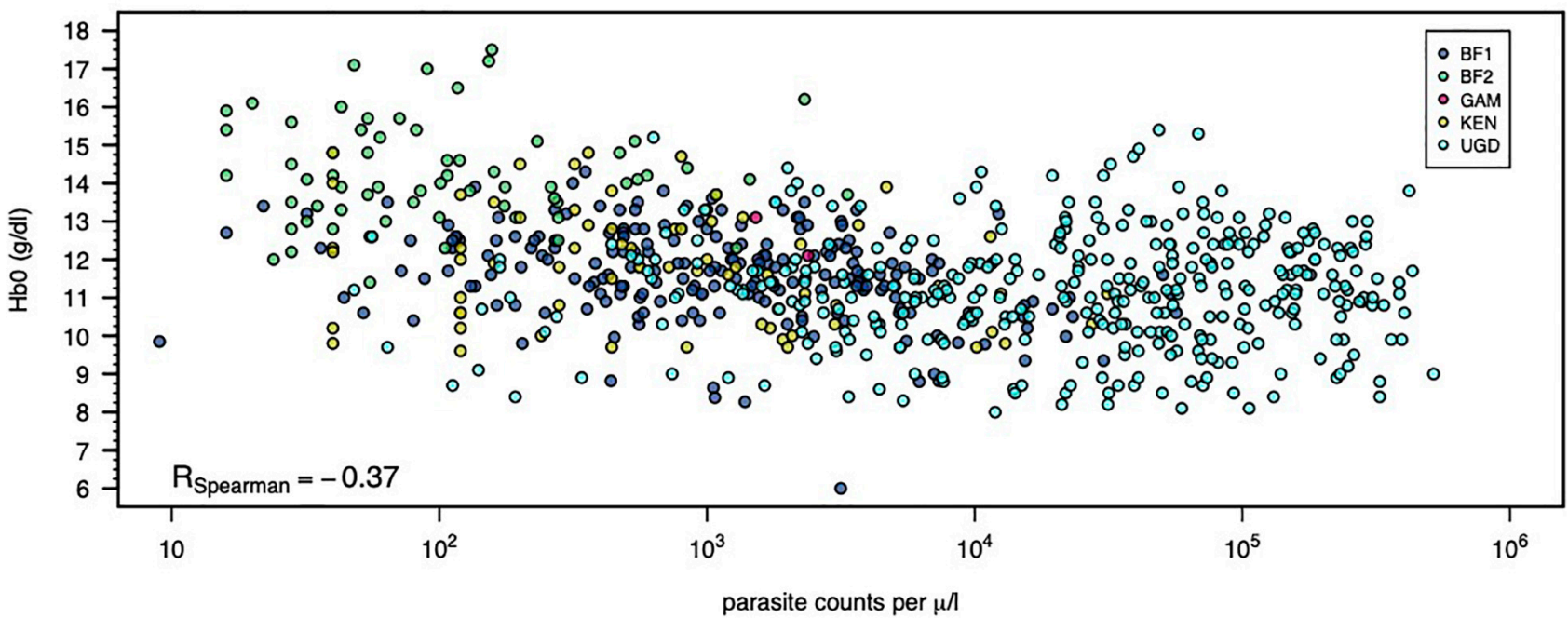

C

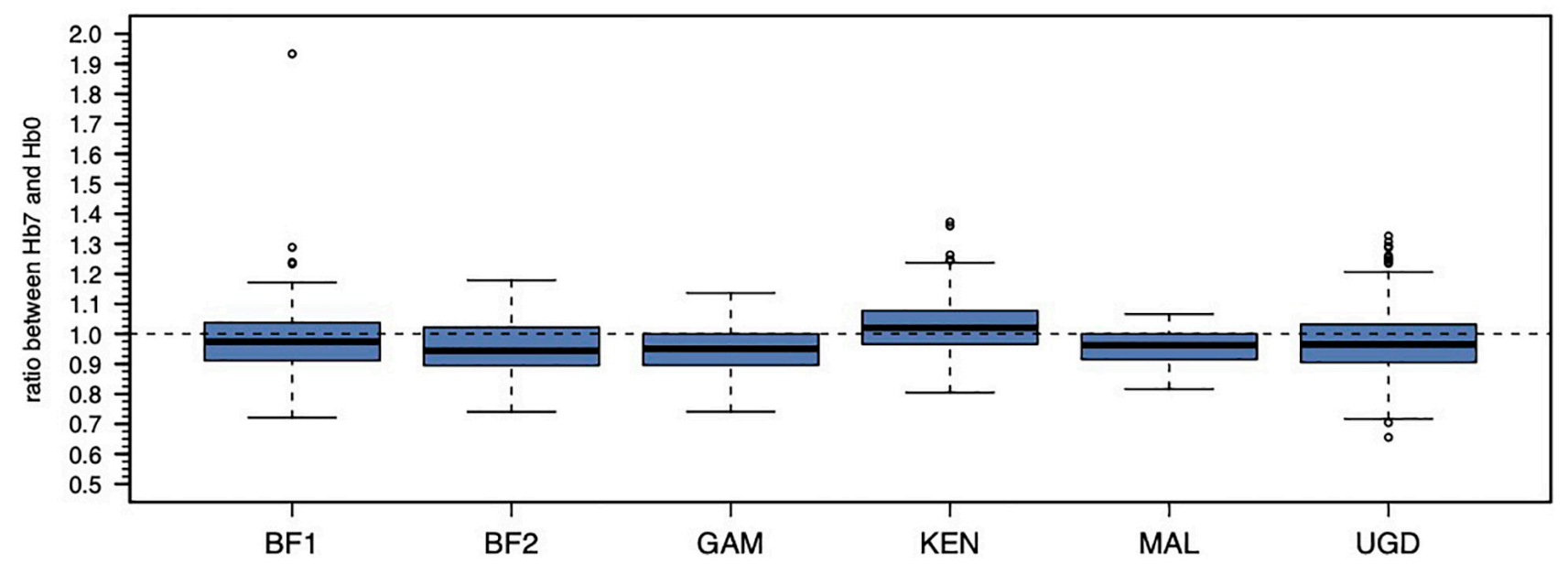

FIGURE 1 | Inter-study variation of $\mathrm{HbO}$ and $\mathrm{Hb}$. (A) Violin plots of $\mathrm{HbO}$ per study. (B) Relationship between $\mathrm{HbO}$ and parasitemia in infected individuals. Note that the study from Mali is not shown because it only recruited non-infected individuals. (C) Violin plots of the ratio between $\mathrm{HbO}$ and $\mathrm{Hb}$. 
TABLE 2 | Frequencies of the reference alleles associated with each of the 20 SNPs located in the G6PD locus defined between 154,326,058 and 154,607,918 positions of $X$ chromosome where the coding region of the gene is located between 154,531,390 and 154,547,569.

\begin{tabular}{|c|c|c|c|c|c|c|c|c|c|c|c|}
\hline $\begin{array}{l}\text { SNP (Associated } \\
\text { Gene) }\end{array}$ & Position & $\begin{array}{l}\text { Functional } \\
\text { consequence }\end{array}$ & Ref $>$ Alt Allele & Gender & BF1 & BF2 & GAM & KEN & MAL & UGD & $1000 G$ \\
\hline \multirow[t]{2}{*}{ rs766420 (TKL1) } & $154,326,058$ & Intron & $C>G$ & Male & 0.355 & N/A & 0.440 & 0.460 & 0.269 & 0.330 & 0.365 \\
\hline & & & & Female & 0.359 & N/A & $\mathrm{N} / \mathrm{A}$ & 0.344 & $N / A$ & 0.361 & \\
\hline rs915941 & $154,398,308$ & Upstream & $A>C$ & Male & 0.591 & N/A & 0.480 & 0.524 & 0.231 & 0.554 & 0.426 \\
\hline (RPL10,SNORA70) & & transcription variant & & Female & 0.500 & $\mathrm{~N} / \mathrm{A}$ & $\mathrm{N} / \mathrm{A}$ & 0.562 & $\mathrm{~N} / \mathrm{A}$ & 0.535 & \\
\hline rs915942 & $154,398,397$ & Upstream & $G>A$ & Male & 0.526 & N/A & 0.667 & 0.607 & 0.846 & 0.606 & 0.588 \\
\hline (RPL10,SNORA70) & & transcription variant & & Female & 0.635 & $\mathrm{~N} / \mathrm{A}$ & $\mathrm{N} / \mathrm{A}$ & 0.562 & $\mathrm{~N} / \mathrm{A}$ & 0.584 & \\
\hline \multirow[t]{2}{*}{ rs28470352 (N/A) } & $154,525,272$ & N/A & $\mathrm{T}>\mathrm{A}$ & Male & 0.702 & $\mathrm{~N} / \mathrm{A}$ & 0.420 & 0.629 & 0.231 & 0.722 & 0.663 \\
\hline & & & & Female & 0.650 & $\mathrm{~N} / \mathrm{A}$ & $\mathrm{N} / \mathrm{A}$ & 0.719 & $\mathrm{~N} / \mathrm{A}$ & 0.671 & \\
\hline \multirow[t]{2}{*}{ rs61042368 (N/A) } & $154,527,122$ & $\mathrm{~N} / \mathrm{A}$ & $G>A$ & Male & 0.751 & $\mathrm{~N} / \mathrm{A}$ & 0.960 & 0.885 & 0.926 & 0.840 & 0.873 \\
\hline & & & & Female & 0.834 & N/A & $\mathrm{N} / \mathrm{A}$ & 0.885 & $\mathrm{~N} / \mathrm{A}$ & 0.894 & \\
\hline \multirow[t]{2}{*}{ rs12389569 (N/A) } & $154,529,519$ & $\mathrm{~N} / \mathrm{A}$ & $G>A$ & Male & 0.900 & N/A & 0.714 & 0.902 & 0.808 & 0.978 & 0.903 \\
\hline & & & & Female & 0.886 & N/A & $\mathrm{N} / \mathrm{A}$ & 0.958 & $\mathrm{~N} / \mathrm{A}$ & 0.938 & \\
\hline rs77214077 & $154,532,214$ & Coding & $G>A$ & Male & 0.953 & $\mathrm{~N} / \mathrm{A}$ & 0.960 & 0.903 & 0.923 & 0.861 & 0.910 \\
\hline$(G 6 P D)$ & & Synonymous & & Female & 0.947 & $\mathrm{~N} / \mathrm{A}$ & $\mathrm{N} / \mathrm{A}$ & 0.854 & $\mathrm{~N} / \mathrm{A}$ & 0.902 & \\
\hline \multirow[t]{2}{*}{ rs2230037 (G6PD) } & $154,532,439$ & Coding, & $G>A$ & Male & 0.674 & 0.861 & 0.800 & 0.661 & 0.962 & 0.766 & 0.714 \\
\hline & & Synonymous & & Female & 0.667 & $\mathrm{~N} / \mathrm{A}$ & $\mathrm{N} / \mathrm{A}$ & 0.698 & $\mathrm{~N} / \mathrm{A}$ & 0.660 & \\
\hline rs73573478 & $154,533,349$ & Intron & $G>A$ & Male & 0.749 & 0.987 & 0.960 & 0.885 & 0.962 & 0.835 & 0.875 \\
\hline$(G 6 P D)$ & & & & Female & 0.833 & $\mathrm{~N} / \mathrm{A}$ & $\mathrm{N} / \mathrm{A}$ & 0.875 & $\mathrm{~N} / \mathrm{A}$ & 0.894 & \\
\hline \multirow[t]{2}{*}{ rs2515905 (G6PD) } & $154,533,860$ & Intron & $G>A$ & Male & 0.888 & 0.351 & 0.780 & 0.839 & 0.481 & 0.833 & 0.827 \\
\hline & & & & Female & 0.838 & $\mathrm{~N} / \mathrm{A}$ & $\mathrm{N} / \mathrm{A}$ & 0.833 & $\mathrm{~N} / \mathrm{A}$ & 0.799 & \\
\hline \multirow[t]{2}{*}{ rs2515904 (G6PD) } & $154,534,556$ & Intron (pathogenic) & $G>C$ & Male & 0.889 & 0.377 & 0.780 & 0.836 & 0.462 & 0.833 & 0.827 \\
\hline & & & & Female & 0.838 & $\mathrm{~N} / \mathrm{A}$ & $\mathrm{N} / \mathrm{A}$ & 0.833 & $\mathrm{~N} / \mathrm{A}$ & 0.796 & \\
\hline rs1050829a,b & $154,535,277$ & Missense variant & $\mathrm{T}>\mathrm{C}$ & Male & 0.694 & 0.234 & 0.420 & 0.629 & 0.192 & 0.722 & 0.662 \\
\hline$(G 6 P D)$ & & & & Female & 0.625 & $\mathrm{~N} / \mathrm{A}$ & $\mathrm{N} / \mathrm{A}$ & 0.719 & N/A & 0.668 & \\
\hline \multirow[t]{2}{*}{ rs1050828 $(G 6 P D)$} & $154,536,002$ & Missense variant & $\mathrm{C}>\mathrm{T}$ & Male & 0.930 & 1.000 & 0.804 & 0.885 & 0.462 & 0.887 & 0.865 \\
\hline & & & & Female & 0.870 & $\mathrm{~N} / \mathrm{A}$ & $\mathrm{N} / \mathrm{A}$ & 0.854 & $\mathrm{~N} / \mathrm{A}$ & 0.872 & \\
\hline \multirow[t]{2}{*}{ rs762515 (G6PD) } & $154,536,313$ & Intron & $\mathrm{T}>\mathrm{C}$ & Male & 0.684 & 0.227 & 0.420 & 0.629 & 0.154 & 0.695 & 0.634 \\
\hline & & & & Female & 0.619 & $\mathrm{~N} / \mathrm{A}$ & $\mathrm{N} / \mathrm{A}$ & 0.708 & $\mathrm{~N} / \mathrm{A}$ & 0.655 & \\
\hline \multirow[t]{2}{*}{ rs762516 (G6PD) } & $154,536,448$ & Intron & $\mathrm{C}>\mathrm{T}$ & Male & 0.888 & 0.355 & 0.780 & 0.836 & 0.462 & 0.833 & 0.827 \\
\hline & & & & Female & 0.838 & $\mathrm{~N} / \mathrm{A}$ & $\mathrm{N} / \mathrm{A}$ & 0.833 & $\mathrm{~N} / \mathrm{A}$ & 0.799 & \\
\hline rs113492957 & $154,544,847$ & Intron & $\mathrm{C} / \mathrm{T}$ & Male & 0.756 & N/A & 0.960 & 0.885 & 0.962 & 0.846 & 0.898 \\
\hline$(G 6 P D, I K B K G)$ & & & & Female & 0.838 & N/A & $\mathrm{N} / \mathrm{A}$ & 0.865 & $\mathrm{~N} / \mathrm{A}$ & 0.894 & \\
\hline \multirow[t]{2}{*}{ rs4898389b (N/A) } & $154,599,376$ & $\mathrm{~N} / \mathrm{A}$ & $G>A$ & Male & 0.977 & N/A & 0.900 & 0.952 & 0.962 & 0.897 & 0.904 \\
\hline & & & & Female & 0.978 & $\mathrm{~N} / \mathrm{A}$ & $\mathrm{N} / \mathrm{A}$ & 0.906 & $\mathrm{~N} / \mathrm{A}$ & 0.935 & \\
\hline \multirow[t]{2}{*}{ rs5986877 b (N/A) } & $154,600,008$ & $\mathrm{~N} / \mathrm{A}$ & $G>C$ & Male & 0.982 & $\mathrm{~N} / \mathrm{A}$ & 0.960 & 0.952 & 0.962 & 0.892 & 0.933 \\
\hline & & & & Female & 0.984 & $\mathrm{~N} / \mathrm{A}$ & $\mathrm{N} / \mathrm{A}$ & 0.896 & $\mathrm{~N} / \mathrm{A}$ & 0.935 & \\
\hline \multirow[t]{2}{*}{ rs7879049b (N/A) } & $154,601,444$ & $\mathrm{~N} / \mathrm{A}$ & $G>A$ & Male & 0.497 & $\mathrm{~N} / \mathrm{A}$ & 0.800 & 0.742 & 0.846 & 0.594 & 0.663 \\
\hline & & & & Female & 0.560 & N/A & $\mathrm{N} / \mathrm{A}$ & 0.604 & $\mathrm{~N} / \mathrm{A}$ & 0.614 & \\
\hline \multirow[t]{2}{*}{ rs60030796 (N/A) } & $154,607,918$ & $\mathrm{~N} / \mathrm{A}$ & $A>G$ & Male & 0.901 & $\mathrm{~N} / \mathrm{A}$ & 0.960 & 0.919 & 0.885 & 0.914 & 0.907 \\
\hline & & & & Female & 0.900 & $N / A$ & N/A & 0.969 & N/A & 0.938 & \\
\hline
\end{tabular}

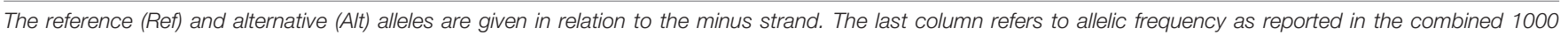
genome project (1000G) for African populations.

a T376C or equivalently A376G.

b Triallelic or quadriallelic SNPS according to reference genome assembly GRCh38.

${ }^{c}$ C202T or equivalently G202A.

The wild type allele of G6PD-deficiency SNP (G202A, rs1050828) was found at a high frequency in most of the studies. The respective relative frequency ranged from $46.2 \%$ (MAL) to $100 \%$ (BF2) in male participants, whilst it was approximately constant in female participants (from $85.4 \%$ in KEN to $87.2 \%$ in UGD). These results are consistent with only recruiting participants with normal G6PD enzymatic activity in most of the studies and, therefore, the respective data was expected to be enriched with wild type alleles of rs 1050828.

\section{Association Analysis Between G6PD SNPs and Hemolysis After PQ Treatment}

Since MAL did not include infected individuals, two separate genetic association analyses related to variation of $\log -\mathrm{Hb} 7$ were 
conducted adjusting for previous confounders but including or not parasitemia at screening as an additional covariate. The strength of association for each SNP was almost indistinguishable adjusting or not for parasitemia. In particular, both analyses suggested putative associations of rs28470352, rs2230037, and rs1050829 (A376G) with log-Hb7 (Figures 3A,B). There was evidence for a very strong LD between rs2230037 and rs1050829 $\left(R^{2}=0.978\right)$. In contrast, these SNPs were in weak LD with rs28470352 ( $R^{2}=0.208$ and 0.227 , respectively). These three polymorphisms were then carried forward to the final stage of the analysis. There was an additional association between rs1050828 (G202A) with the phenotype that was only statistically significant adjusting for confounding but excluding parasitemia (Figure 3A).

The final stage of the association analysis comprised the assessment of the joint effect of the three detected SNPs on the phenotype using extended regression models where genetic and non-genetic effects were contemplated (Table 3). Since there were no data available for rs 28470352 from BF2, two extended models were fitted to the data including or not the effect of that genetic marker; these models were denoted hereafter as $M_{1}$ and $M_{2}$, respectively. As a consequence, the estimation of $\mathrm{M}_{1}$ was based on a smaller sample size $(n=880)$ due to the exclusion of the data from that SNP. In contrast, the estimation of $\mathrm{M}_{2}$ was based on the whole data set $(n=957)$.

Both $M_{1}$ and $M_{2}$ led to estimates for the non-genetic variables similar to the ones obtained in absence of genetic effects (Table 3 ). More importantly, the effects of rs28470352 and rs1050829 were not statistically significant. Positive effects on log-Hb7 were observed for the AA genotype of rs2230037 (coefficient estimates $=0.020$ and 0.018 for $\mathrm{M}_{1}$ and $\mathrm{M}_{2}$, respectively). When transformed into linear scale, these effects predicted an increase of $1.02 \mathrm{~g} / \mathrm{dL}$ in $\mathrm{Hb} 7$ when compared to the GG genotype of rs223037. However, these effects were in the borderline of statistical significance ( $p$-values $=0.041$ and 0.051 , respectively). In conclusion, the effect size and its statistical significance suggested that these genetic markers appear to have a minor role in explaining $\mathrm{Hb} 7$ in relation to non-genetic factors, such as age, parasitemia or $\mathrm{Hb} 0$.

\section{DISCUSSION}

This study aimed to evaluate the effect of genetic polymorphisms within G6PD locus on hemolysis on individuals treated with single doses of $\mathrm{PQ}$. The rationale was to provide information for safety concerns associated with the broader population delivery of PQ given the potentially high prevalence of G6PD deficiency in African populations. This was an opportunistic study, typing G6PD SNPs in retrospective samples collected from completed efficacy or safety of single dose PQ in which Hb data were collected and, as such, there is a number of limitations. Notwithstanding this fact, we identified putative associations of the common A376G variant in African population and two lessknown SNP with Hb7. However, these G6PD polymorphisms appear to have minor effects when compared to the strong nongenetic effects related to age, parasitemia, and $\mathrm{Hb} 0$. This finding is reassuring from the point of drug safety, because it seems to rule out any specific effect of G6PD variants on hemolysis other than the PQ effect alone. In addition, it is important to note that, as described in the original papers, hemolysis was only transient in the study participants who had normal levels of $\mathrm{Hb}$ at the end of the respective trials. Therefore, the drug could be considered safe irrespective of the G6PD variation present in the study participants, as discussed in the original references (Bastiaens et al., 2018; Chen et al., 2018) and in a safety study from Tanzania (Mwaiswelo et al., 2016).

As stated above a limitation is that five out of six studies in our analysis excluded individuals with reduced G6PD enzymatic activity and, therefore, the deleterious allele A in G202A was under-represented in the data through selection bias. This exclusion criterium reduced the probability to detect any putative association between that SNP and Hb7. Previous associations with drops in $\mathrm{Hb}$ and both G202A and A376G have been shown in treatment trials with PQ but this was with the higher dose of $0.75 \mathrm{mg} / \mathrm{kg}$ (Shekalaghe et al., 2009, 2010). Interestingly, the one individual in those studies that had a significant drop in $\mathrm{Hb}$ levels was wild type at both SNPs suggesting involvement of other genes elsewhere in the genome, which was in part the motivation for this study.

We addressed further differences between studies by adjusting for age and gender in our genetic association analysis. In addition, age and gender could also control for possible confounding on the dynamics of $\mathrm{Hb}$ during treatment and these were assumed to be independent of other variables analyzed. A study-specific effect was also included in the linear regression models to capture latent (genetic) differences between populations and potential differences in research protocols. Interestingly, all studies but KEN had similar study-specific effects in the data. This suggests that the level of hemolysis observed at day 7 was fairly consistent across studies. These study-specific effects together with the effects of parasitemia and $\mathrm{HbO}$ explained more than half of the variation observed in $\mathrm{Hb} 7$ and suggested that the remaining variation is not explained by the genetic variation in the G6PD locus. Further variation might be due to further individual variation in the rate by which the drug is metabolized in the body. Genetic polymorphisms on CYP2D6 gene, which are known to influence the rate by which PQ is metabolized in the body (Gonçalves et al., 2017; Saito et al., 2018), have been associated with variation in post treatment gametocyte carriage using data from 8 clinical trials including the ones here analyzed (Pett et al., 2019), but a high proportion of missing CYP2D6 data precluded a more integrated genetic association analysis including the joint effects of G6PD and CYP2D6 allelic variants on hemolysis.

The KEN study was identified as having a higher studyspecific effect than the remaining studies. This study was the only one where G6PD deficiency status was not determined in the participants at enrollment (Stone et al., 2017). However, the frequency of the deleterious allele A associated with G202A was consistent with the other studies.

Our study comprised a relatively small number of genotyped SNPs when compared to more than 250 genetic variants already identified within the G6PD locus (Clarke et al., 2017). Despite this hypothetical large number of genetic variants in the G6PD 
A

\section{Comparison between studies}

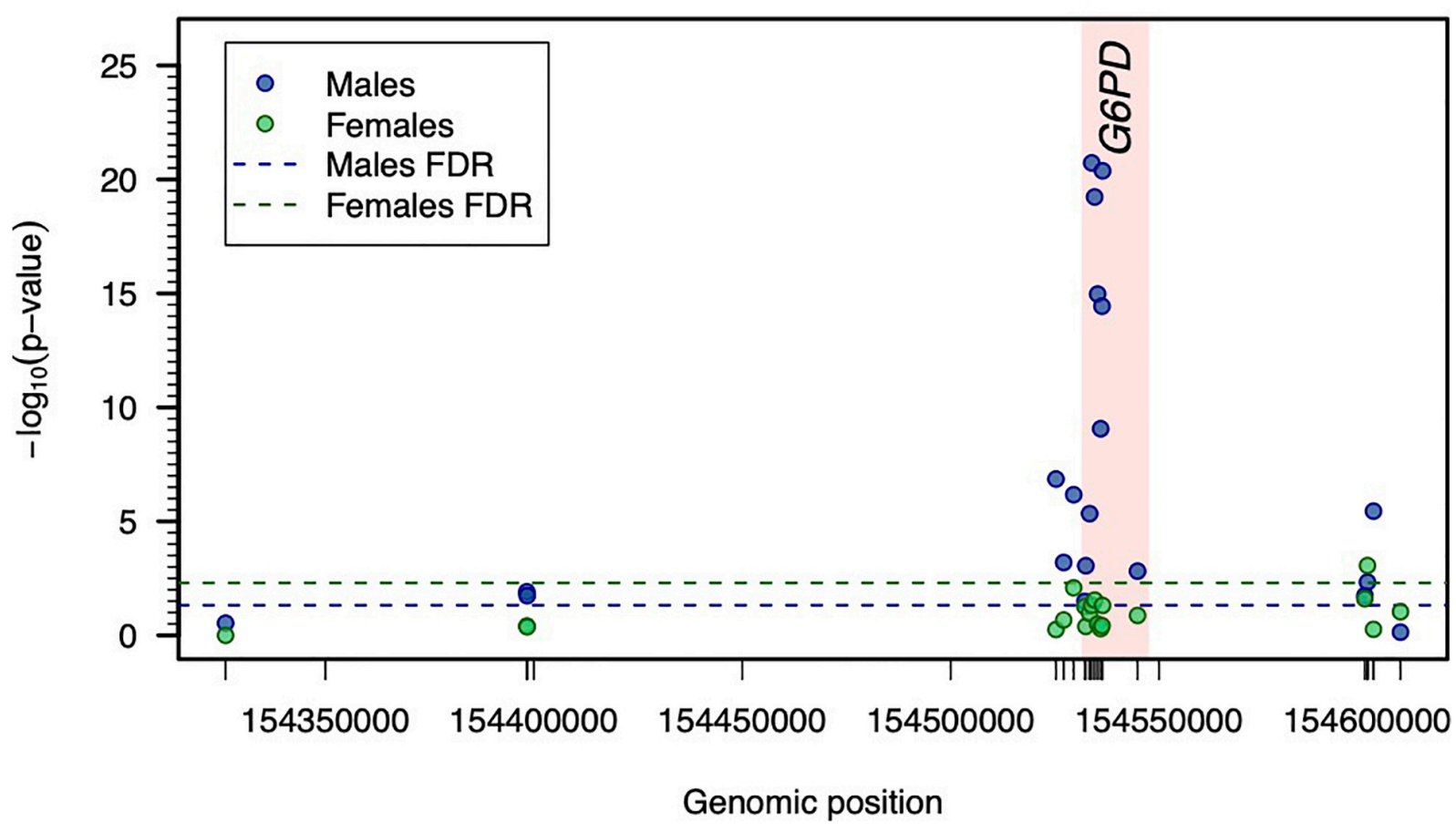

B Comparison to 1000 Genome data

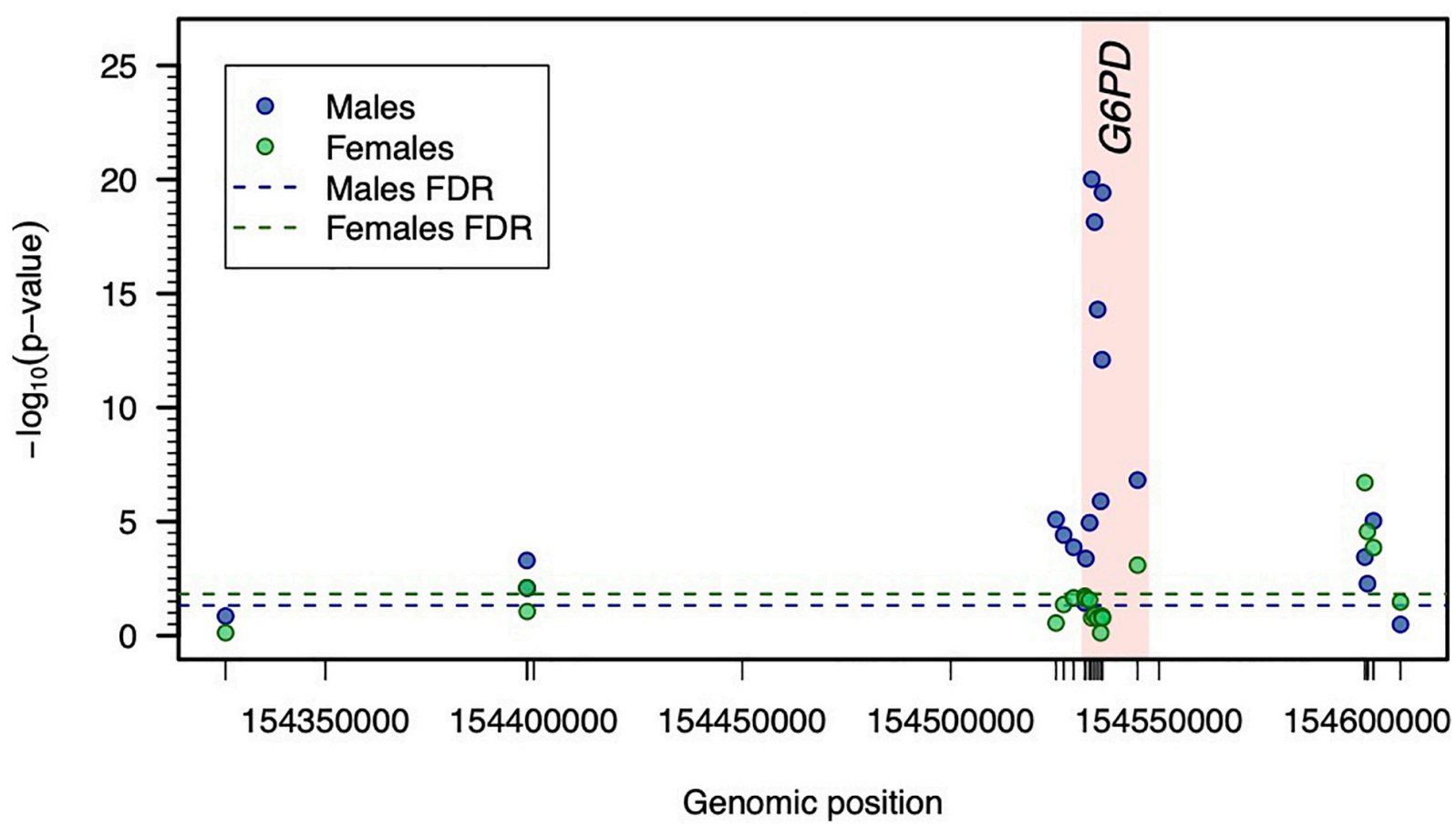

FIGURE 2 | Inter-study variation with respect to the genetic data. (A) Comparison of genotype frequencies between studies. Statistical significance was determined by $-\log _{10}(p$-value) associated with the Pearson's test for independence in two-way frequency tables. For males and females, the thresholds for statistical significance were calculated separately and were determined to ensure a false discovery rate (FDR) of $5 \%$. (B) Comparison between allelic frequencies between studies and 1000 Genome Project data. The reported - $\log _{10}(p$-value) refers to the minimum $p$-value of Binomial test for comparing the allelic frequency of a given SNP in each study assuming the respective allelic frequency of 1000G project data as the one under the null hypothesis (see Table 2). 
A

Not controlling for parasitaemia

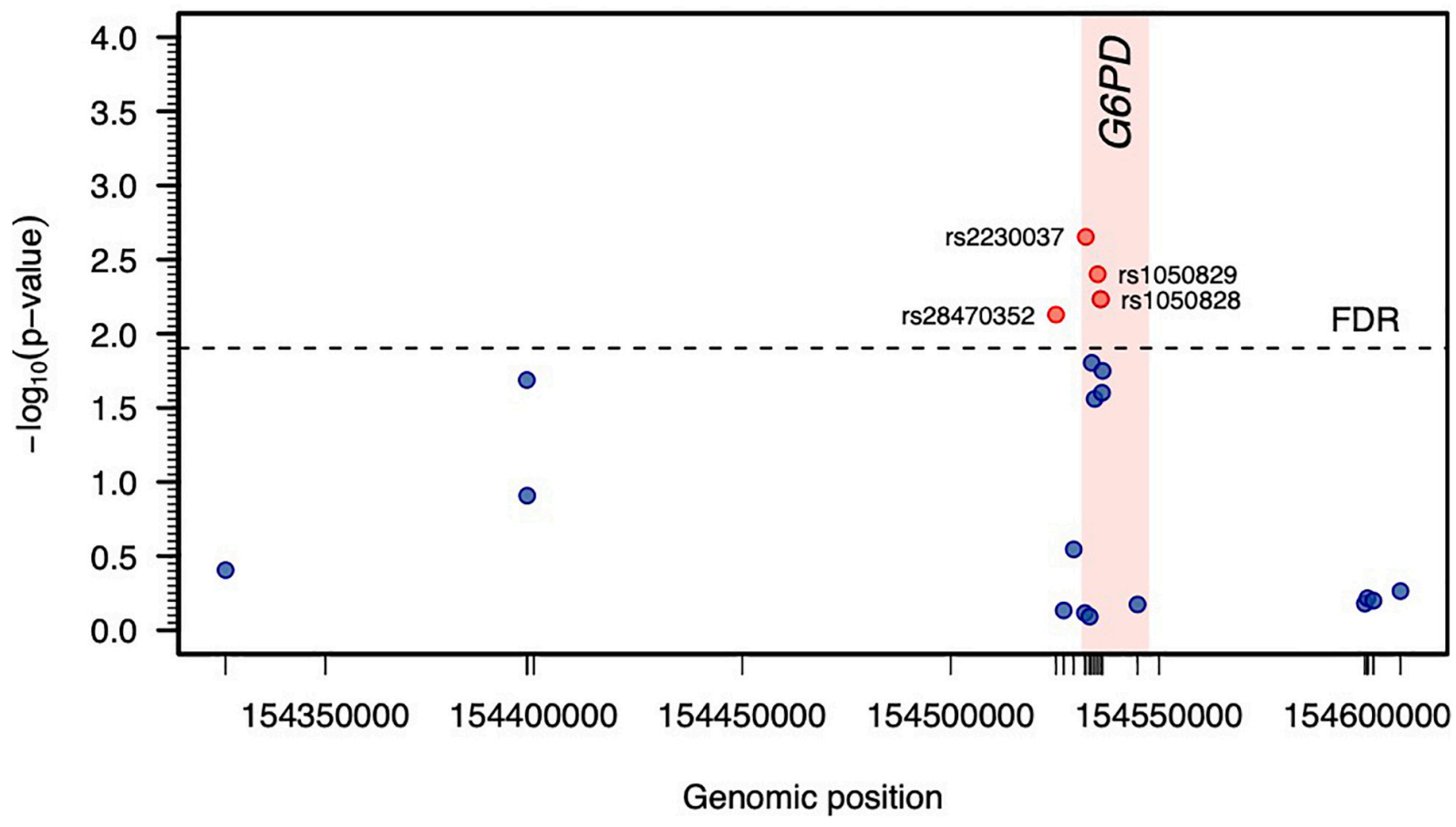

B

Controlling for parasitaemia

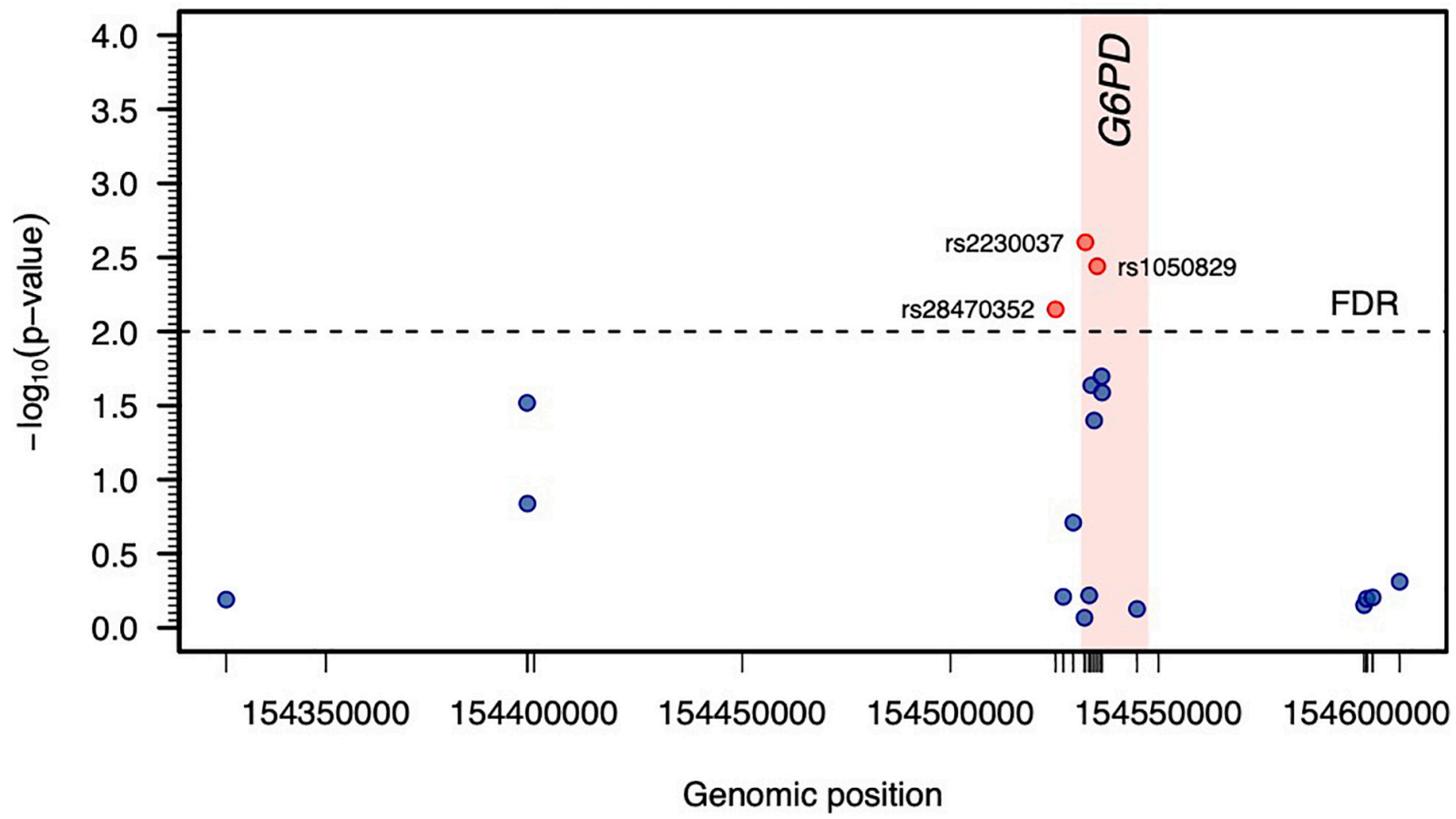

FIGURE 3 | Genetic association analysis between log-Hb7 and each SNP adjusting or not for parasitemia at day 0 (A,B, respectively). Statistical significance was determined by the maximum of $-\log _{10}(p$-value) associated with the log-likelihood ratio tests when testing different genetic effects of each SNP in a linear regression model adjusting for age, gender, parasitemia at screening, and log- $\mathrm{HbO}$. The threshold for statistical significance was determined to ensure a false discovery rate (FDR) of $5 \%$. 
TABLE 3 | Estimation of the joint genetic models $M_{1}$ (excluding data from BF2) and $\mathrm{M}_{2}$ (based on the whole data set) for the association between log- $\mathrm{Hb} 7$ and the best G6PD SNP adjusting or not for parasitemia at day 0 where the alleles $A$ and $\mathrm{B}$ denote the reference and alternative alleles shown in Table $\mathbf{2}$.

\begin{tabular}{|c|c|c|c|c|}
\hline \multirow[b]{2}{*}{ Predictor } & \multicolumn{2}{|c|}{ Model $\mathbf{M}_{1}$} & \multicolumn{2}{|c|}{ Model $\mathbf{M}_{2}$} \\
\hline & Estimate (SE) & $P$-value & Estimate (SE) & $P$-value \\
\hline Intercept & $1.021(0.068)$ & $<0.001$ & $1.020(0.066)$ & $<0.001$ \\
\hline \multicolumn{5}{|l|}{ Study } \\
\hline BF1 (reference) & - & - & - & - \\
\hline $\mathrm{BF} 2$ & $\mathrm{NA}(\mathrm{NA})$ & $\mathrm{NA}$ & $-0.015(0.021)$ & 0.463 \\
\hline GAM & -0.019 (0.019) & 0.327 & -0.014 (0.019) & 0.442 \\
\hline KEN & $0.047(0.011)$ & $<0.001$ & $0.047(0.011)$ & $<0.001$ \\
\hline MAL & -0.018 (0.033) & 0.577 & -0.004 (0.029) & 0.903 \\
\hline UGD & $0.015(0.009)$ & 0.100 & $0.012(0.009)$ & 0.159 \\
\hline Age (per year) & $0.005(0.001)$ & $<0.001$ & $0.004(0.001)$ & $<0.001$ \\
\hline \multicolumn{5}{|l|}{$P Q$ dose $(\mathrm{mg} / \mathrm{kg})$} \\
\hline 0 & - & - & - & - \\
\hline $0.10-0.40$ & -0.007 (0.008) & 0.399 & -0.007 (0.008) & 0.328 \\
\hline $0.41-0.75$ & $-0.016(0.012)$ & 0.175 & -0.017 (0.012) & 0.159 \\
\hline \multicolumn{5}{|l|}{ Gender } \\
\hline Female (reference) & - & - & - & - \\
\hline Male & $-0.010(0.009)$ & 0.261 & -0.012 (0.009) & 0.193 \\
\hline $\log -\mathrm{HbO}$ & $0.561(0.029)$ & $<0.001$ & $0.565(0.027)$ & $<0.001$ \\
\hline Parasitemia (x 100,000) & $-0.033(0.005)$ & $<0.001$ & $-0.033(0.005)$ & $<0.001$ \\
\hline \multicolumn{5}{|l|}{ rs28470352 } \\
\hline$\Pi \mathrm{T}$ (reference) & - & - & - & - \\
\hline TA & $-0.003(0.040)$ & 0.946 & - & - \\
\hline$A A$ & $-0.061(0.065)$ & 0.353 & - & - \\
\hline \multicolumn{5}{|l|}{ rs2230037 } \\
\hline GG (reference) & - & - & - & - \\
\hline GA & $0.007(0.010)$ & 0.467 & $0.006(0.010)$ & 0.506 \\
\hline AA & $0.020(0.010)$ & 0.041 & $0.018(0.009)$ & 0.051 \\
\hline \multicolumn{5}{|l|}{ rs1050829 } \\
\hline Tा (reference) & - & - & - & - \\
\hline CT & $-0.012(0.040)$ & 0.768 & $-0.016(0.010)$ & 0.117 \\
\hline $\mathrm{CC}$ & $0.051(0.065)$ & 0.439 & $-0.011(0.009)$ & 0.208 \\
\hline
\end{tabular}

locus, many of catalogued SNPs have too-low minor allele or they are only present in non-African populations. In this regard, the evaluated SNPs were considered the most informative in terms of allele frequency in African populations. As a limitation, half of the evaluated SNPs were outside the G6PD coding region. Given this scenario, it is then possible that additional association signals could derived from rare genetic variants located in this locus. However, these association signals could only be detected in studies with large sample sizes, as illustrated by the UK10k consortium (Walter et al., 2015) and the study of genetic variants related to blood pressure (Surendran et al., 2020). It is worth noting that the telomeric location of the G6PD gene on the $\mathrm{X}$ chromosome imposes serious limitations in terms of the assay costs and scalability to a large number of samples and SNP under analysis, as illustrated in the most comprehensive genetic study of G6PD variation conducted so far (Clarke et al., 2017). From an initial number of more than 250 SNPs, the authors considered the study only feasible in a benchmark set of 65
SNPs located in a $560 \mathrm{~kb}$ region spanning the G6PD locus itself and part of the overlapping $I K B K B$ gene (encoding Inhibitor of Kappa Light Polypeptide Gene Enhancer in B-Cells). Since our study comprised the analysis of retrospective data, there was a limitation to the number of SNP under analysis. In this regard, we considered these SNPs to be a good representation of this reference set, because only three SNPs were discarded after our quality control checks.

Despite the inherent limitations in attempting to combine different studies, all used a single dose of PQ, had measures of $\mathrm{Hb} 7$ after the initiation of treatment and the same core group of G6PD-associated SNPs were tested. Consistent with previous reports we reported borderline associations of specific G6PD SNPs with mild transient hemolysis. This provides further evidence that single, low dose PQ can be deployed in larger population studies.

\section{DATA AVAILABILITY STATEMENT}

The raw data supporting the conclusion of this article will be made available by the authors, without undue reservation.

\section{ETHICS STATEMENT}

The studies involving human participants were reviewed and approved by the Ethics Committee of the Faculty of Medicine, Pharmacy, and Dentistry, University of Science, Techniques and Technologies of Bamako, and the Committee on Human Research at the University of California, San Francisco (studies in Mali); the Comité d'Ethique pour la Recherche en Santé, Ministère de la Santé du Burkina Faso, and the Comité Technique d'Examen des Demandes d'Autorisation d'Essais Cliniques, Ministère de la Santé du Burkina Faso (studies in Burkina Faso); the Gambia Government/MRC Joint Ethics Committee (studies in the Gambia); the Makerere University School of Medicine Research Ethics Committee and the Uganda National Council of Science and Technology (study in Uganda); the Kenya Medical Research Institute Ethics Review Committee (study in Kenya); and the Interventions Research Ethics Committee of the London School of Hygiene and Tropical Medicine (all studies). Written informed consent to participate in this study was provided by the participants' legal guardian/next of kin.

\section{AUTHOR CONTRIBUTIONS}

TB and CD conceived the research. JC, LM, GB, AT, JO, SAC, BG, MA, AO, EB, GS, IN, KL, SS, AD, Ud'A, IC, AE, RG, TB, and $\mathrm{CD}$ were involved in data collection, lab processing, and data interpretation of the clinical trials analyzed. NS, LG, JC, TC, and SC decided the list of SNPs to be genotyped. LG, JC, and SC organized and performed the SNP genotyping. NS performed the statistical analysis. NS, LG, and CD wrote the manuscript. All authors have revised, read, and approved the final version of the manuscript. 


\section{FUNDING}

TC was funded by the Medical Research Council UK (Grant no. MR/M01360X/1, MR/N010469/1, MR/R025576/1, and MR/R020973/1) and BBSRC UK (Grant no. BB/R013063/1). SC was funded by Bloomsbury SET, Medical Research Council UK (MR/M01360X/1, MR/R025576/1, and MR/R020973/1), and BBSRC UK (Grant no. BB/R013063/1). AE received funding from a Wellcome Clinical Fellowship (090558). LG, BG, and $\mathrm{CD}$ were funded from the Bill \& Melinda Gates Foundation

\section{REFERENCES}

Auton, A., Abecasis, G. R., Altshuler, D. M., Durbin, R. M., Bentley, D. R., Chakravarti, A., et al. (2015). A global reference for human genetic variation. Nature 526, 68-74. doi: 10.1038/nature15393

Awandu, S. S., Raman, J., Makhanthisa, T. I., Kruger, P., Frean, J., Bousema, T., et al. (2018). Understanding human genetic factors influencing primaquine safety and efficacy to guide primaquine roll-out in a pre-elimination setting in southern Africa. Malar. J. 17:120. doi: 10.1186/s12936-0182271-Z

Baird, J. K., and Surjadjaja, C. (2011). Consideration of ethics in primaquine therapy against malaria transmission. Trends Parasitol. 27, 11-16. doi: 10.1016/ j.pt.2010.08.005

Bastiaens, G. J. H., Tiono, A. B., Okebe, J., Pett, H. E., Coulibaly, S. A., Gonçalves, B. P., et al. (2018). Safety of single low-dose primaquine in glucose-6-phosphate dehydrogenase deficient falciparum-infected African males: two open-label, randomized, safety trials. PLoS One 13:e0190272. doi: 10.1371/journal.pone. 0190272

Benjamini, Y., and Hochberg, Y. (1995). Controlling the false discovery rate: a practical and powerful approach to multiple testing. J. R. Stat. Soc. Series B Stat. Methodol. 57, 289-300.

Beutler, E., Kuhl, W., Vives-Corrons, J., and Prchal, J. (1989). Molecular heterogeneity of glucose-6-phosphate dehydrogenase A-. Blood 74, 2550-2555. doi: 10.1182/blood.v74.7.2550.2550

Chen, I., Diawara, H., Mahamar, A., Sanogo, K., Keita, S., Kone, D., et al. (2018). Safety of single-dose primaquine in G6PD-deficient and G6PD-normal males in mali without malaria: an open-label, phase 1, dose-adjustment trial. J. Infect. Dis. 217, 1298-1308. doi: 10.1093/infdis/jiy014

Clarke, G. M., Rockett, K., Kivinen, K., Hubbart, C., Jeffreys, A. E., Rowlands, K., et al. (2017). Characterisation of the opposing effects of G6PD deficiency on cerebral malaria and severe malarial anaemia. Elife 6:e15085.

Dicko, A., Brown, J. M., Diawara, H., Baber, I., Mahamar, A., Soumare, H. M., et al. (2016). Primaquine to reduce transmission of Plasmodium falciparum malaria in Mali: a single-blind, dose-ranging, adaptive randomised phase 2 trial. Lancet Infect. Dis. 16, 674-684. doi: 10.1016/S1473-3099(15) 00479-X

Eziefula, A. C., Bousema, T., Yeung, S., Kamya, M., Owaraganise, A., Gabagaya, G., et al. (2014). Single dose primaquine for clearance of Plasmodium falciparum gametocytes in children with uncomplicated malaria in Uganda: a randomised, controlled, double-blind, dose-ranging trial. Lancet Infect. Dis. 14, 130-139. doi: 10.1016/S1473-3099(13)70268-8

Gonçalves, B. P., Pett, H., Tiono, A. B., Murry, D., Sirima, S. B., Niemi, M., et al. (2017). Age, weight, and CYP2D6 genotype are major determinants of primaquine pharmacokinetics in African children. Antimicrob. Agents Chemother. 61:e02590-16. doi: 10.1128/AAC.02590-16

Gonçalves, B. P., Tiono, A. B., Ouédraogo, A., Guelbéogo, W. M., Bradley, J., Nebie, I., et al. (2016). Single low dose primaquine to reduce gametocyte carriage and Plasmodium falciparum transmission after artemetherlumefantrine in children with asymptomatic infection: a randomised, double-blind, placebo-controlled trial. BMC Med. 14:40. doi: 10.1186/s12916-016-0581-y

Grabowska, A. D., Lacerda, E. M., Nacul, L., and Sepúlveda, N. (2020). Review of the quality control checks performed by current genome-wide for the Primaquine supplement to AFIRM (OPP1034789). NS was partially funded by FCT - Fundação para a Ciência e a Tecnologia, Portugal (Ref. Grant: UIDB/00006/2020).

\section{SUPPLEMENTARY MATERIAL}

The Supplementary Material for this article can be found online at: https://www.frontiersin.org/articles/10.3389/fgene. 2021.645688/full\#supplementary-material

and targeted-genome association studies on myalgic encephalomyelitis/chronic fatigue syndrome. Front. Pediatr. 8:293. doi: 10.3389/fped.2020.00293

Hirono, A., and Beutler, E. (1988). Molecular cloning and nucleotide sequence of cDNA for human glucose-6-phosphate dehydrogenase variant A(-). Proc. Natl. Acad. Sci. U. S. A. 85, 3951-3954. doi: 10.1073/pnas.85.11.3951

Howes, R. E., Piel, F. B., Patil, A. P., Nyangiri, O. A., Gething, P. W., Dewi, M., et al. (2012). G6PD deficiency prevalence and estimates of affected populations in malaria endemic countries: a geostatistical model-based map. PLoS Med. 9:e1001339. doi: 10.1371/journal.pmed.1001339

Maiga, B., Dolo, A., Campino, S., Sepulveda, N., Corran, P., Rockett, K. A., et al. (2014). Glucose-6-phosphate dehydrogenase polymorphisms and susceptibility to mild malaria in Dogon and Fulani, Mali. Malar. J. 13:270. doi: 10.1186/14752875-13-270

Manjurano, A., Sepulveda, N., Nadjm, B., Mtove, G., Wangai, H., Maxwell, C., et al. (2015). African glucose-6-phosphate dehydrogenase alleles associated with protection from severe malaria in heterozygous females in Tanzania. PLoS Genet. 11:e1004960. doi: 10.1371/journal.pgen.1004960

Mwaiswelo, R., Ngasala, B. E., Jovel, I., Gosling, R., Premji, Z., Poirot, E., et al. (2016). Safety of a single low-dose of primaquine in addition to standard artemether-lumefantrine regimen for treatment of acute uncomplicated Plasmodium falciparum malaria in Tanzania. Malar. J. 15:316. doi: 10.1186/ s12936-016-1341-3

Pett, H., Bradley, J., Okebe, J., Dicko, A., Tiono, A. B., Gonçalves, B. P., et al. (2019). CYP2D6 polymorphisms and the safety and gametocytocidal activity of singledose primaquine for Plasmodium falciparum. Antimicrob. Agents Chemother. 63:e00538-19. doi: 10.1128/AAC.00538-19

Rockett, K. A., Clarke, G. M., Fitzpatrick, K., Hubbart, C., Jeffreys, A. E., Rowlands, K., et al. (2014). Reappraisal of known malaria resistance loci in a large multicenter study. Nat. Genet. 46, 1197-1204.

Saito, T., Gutiérrez Rico, E. M., Kikuchi, A., Kaneko, A., Kumondai, M., Akai, F., et al. (2018). Functional characterization of 50 CYP2D6 allelic variants by assessing primaquine 5-hydroxylation. Drug Metab. Pharmacokinet. 33, 250-257. doi: 10.1016/j.dmpk.2018.08.004

Sepúlveda, N., Manjurano, A., Campino, S. G., Lemnge, M., Lusingu, J., Olomi, R., et al. (2017). Malaria host candidate genes validated by association with current, recent, and historical measures of transmission intensity. J. Infect. Dis. 216, 45-54.

Shekalaghe, S., Alifrangis, M., Mwanziva, C., Enevold, A., Mwakalinga, S., Mkali, H., et al. (2009). Low density parasitaemia, red blood cell polymorphisms and Plasmodium falciparum specific immune responses in a low endemic area in northern Tanzania. BMC Infect. Dis. 9:69. doi: 10.1186/1471-23 34-9-69

Shekalaghe, S. A., ter Braak, R., Daou, M., Kavishe, R., Van den Bijllaardt, W., Van den Bosch, S., et al. (2010). In Tanzania, hemolysis after a single dose of primaquine coadministered with an artemisinin is not restricted to glucose-6phosphate dehydrogenase-deficient (G6PD A-) individuals. Antimicrob. Agents Chemother. 54, 1762-1768. doi: 10.1128/AAC.01135-09

Stone, W., Sawa, P., Lanke, K., Rijpma, S., Oriango, R., Nyaurah, M., et al. (2017). A molecular assay to quantify male and female Plasmodium falciparum gametocytes: results from 2 randomized controlled trials using primaquine for gametocyte clearance. J. Infect. Dis. 216, 457-467. doi: 10.1093/infdis/ jix237 
Surendran, P., Feofanova, E. V., Lahrouchi, N., Ntalla, I., Karthikeyan, S., Cook, J., et al. (2020). Discovery of rare variants associated with blood pressure regulation through meta-analysis of 1.3 million individuals. Nat. Genet. 52, 1314-1332. doi: 10.1038/s41588-020-00713-x

Walter, K., Min, J. L., Huang, J., Crooks, L., Memari, Y., McCarthy, S., et al. (2015). The UK10K project identifies rare variants in health and disease. Nature 526, 82-89. doi: 10.1038/nature14962

WHO Malaria Policy Advisory Committee and Secretariat (2012). Malaria Policy Advisory Committee to the WHO: conclusions and recommendations of September 2012 meeting. Malar. J. 11:424. doi: 10.1186/1475-2875$11-424$

World Health Organization (2012). Updated WHO Policy Recommendation: Single Dose Primaquine as a Gametocytocide in Plasmodium Falciparum Malaria. Available online at: https://www.who.int/malaria/publications/atoz/who_pq policy_recommendation/en/ (accessed November 29, 2020)
Conflict of Interest: JC and LM were employed by the company LGC Genomics.

The remaining authors declare that the research was conducted in the absence of any commercial and financial relationships that could be construed as a potential conflict of interest.

Copyright (๑) 2021 Sepúlveda, Grignard, Curry, Mahey, Bastiaens, Tiono, Okebe, Coulibaly, Gonçalves, Affara, Ouédraogo, Bougouma, Sanou, Nébié, Lanke, Sirima, Dicko, d'Alessandro, Clark, Campino, Chen, Eziefula, Gosling, Bousema and Drakeley. This is an open-access article distributed under the terms of the Creative Commons Attribution License (CC BY). The use, distribution or reproduction in other forums is permitted, provided the original author $(s)$ and the copyright owner $(s)$ are credited and that the original publication in this journal is cited, in accordance with accepted academic practice. No use, distribution or reproduction is permitted which does not comply with these terms. 